\title{
REFERENCES
}

1. J. H. Mulligan, Jr., The effect of pole and zero locations on the transient response of linear, dynamic systems, Proc. I. R. E. vol. 37 (1949) pp. 516-529.

2. E. Lukacs and O. Szász, Certain Fourier transforms of distributions, Canad. J. Math. vol. 3 (1951) pp. 140-144.

3. - Certain Fourier transforms of distributions: II, Canad. J. Math. vol. 6 (1954) pp. 186-189.

4. K. Takano, Certain Fourier transforms of distributions, Tôhoku Math J. (2) vol. 3 (1951) pp. 306-315.

5. E. Lukacs and O. Szász, Some non-negative trigonometric polynomials connected with a problem in probability, J. Research N.B.S. vol. 48 (1952) pp. 139-146.

6. - Non-negative trigonometric polynomials and certain rational characteristic functions, J. Research N.B.S. vol. 52 (1954) pp. 153-160. 1946.

7. D. V. Widder, The Laplace transform, Princeton, Princeton University Press,

8. M. R. Aaron and R. G. Segers, A necessary and sufficient condition for a bounded nondecreasing step response, I. R. E. Transactions vol. CT-5 (1958) pp. 226-227.

NEW YORK UNIVERSITY

\section{ON PROJECTIONS OF SEPARABLE SUBSPACES OF $(m)$ ONTO $(c)$}

\section{R. D. MCWILLIAMS ${ }^{1}$}

1. There exists no bounded projection of the Banach space $(m)$ of bounded real sequences onto the space $(c)$ of convergent sequences [1] or onto the space $\left(c_{0}\right)$ of null sequences [2]. It has been shown however by Sobczyk [2] that if $B$ is a separable subspace of $(m)$ properly containing $\left(c_{0}\right)$, then there exists a projection $P$ of norm 2 of $B$ onto $\left(c_{0}\right)$.

In the present paper we show that if $B$ is a separable subspace of $(m)$ containing $(c)$, then there is a projection $Q$ of $B$ onto $(c)$ with $\|Q\| \leqq 3$. We then prove a theorem giving a lower bound for the norms of projections onto $(c)$ of spaces of the form $(c)+(x)$, where $(x)$ is the one-dimensional subspace determined by an element $x \notin(c)$. Using this result, we exhibit for each $n>1$ a subspace $B_{n}$ determined by $(c)$ and $n-1$ other elements of $(m)$, such that the minimum of the norms of projections of $B_{n}$ onto $(c)$ is $3-2 n^{-1}$. It follows that there exists a separable subspace $B \supset(c)$ such that any projection of $B$ onto $(c)$ has norm at least 3 .

Received by the editors February 16, 1959.

${ }^{1}$ Some of the results of this paper were contained in the author's University of Tennessee thesis and were obtained during the tenure of a National Science Foundation fellowship, and some were obtained under Army Office of Ordnance Research Contract \#DA-36-034-ORD-2001, OOR Project \#264. 
2. The following lemma adds to Sobczyk's theorem [2, pp. 942944] the fact that if $B$ contains (c), then $P$ can be chosen so that $P e=0$, where $e=(1,1, \cdots)$.

Lemma 2.1. If $B$ is a separable subspace of $(m)$ containing $(c)$, then there exists a projection $P$ of $B$ onto $\left(c_{0}\right)$ such that $\|P\|=2$ and $P e=0$.

For the proof of the lemma, we follow Sobczyk's proof ${ }^{2}$ in every detail except that we can and do take the first element $X_{1}$ of the sequence $X_{1}, X_{2}, \cdots$ to be $e$. It then follows that $P^{N} e=0$ for each $N$ and hence that the limit $P e=\lim _{N} P^{N} e=0$.

Theorem 2.2. If $B$ is a separable subspace of $(m)$ containing $(c)$, then there exists a projection $Q$ of $B$ onto $(c)$ such that $\|Q\| \leqq 3$.

Proof. Let $f(y)=\lim _{j} y_{j}$ for all $y=\left(y_{j}\right) \in(c)$. Then $f \in(c)^{*}$ and $\|f\|=1$, and by the Hahn-Banach theorem there exists an $F \in B^{*}$ such that $\|F\|=1$ and $F$ is an extension of $f$. Define

$$
Q x=P x+F(x) e,
$$

where $P$ is a projection of $B$ onto $\left(c_{0}\right)$ with $\|P\|=2$ and $P e=0$, as given by Lemma 2.1. Then $Q$ is a projection of $B$ onto $(c)$, and $\|Q\| \leqq\|P\|+\|F\|=3$.

3. In the present section we consider subspaces of the form $B=(c)+(x)$ where $x=\left(x_{j}\right)$ is in $(m)$ but not in $(c)$.

Theorem 3.1. Let $x$ be an element of $(m)$ such that $\alpha>\beta \geqq-\alpha$, where $\alpha=\lim \sup _{j} x_{j}$ and $\beta=\lim \inf _{j} x_{j}$. If $P$ is any projection of $(c)+(x)$ onto (c) such that $P x=\left(y_{j}\right) \in\left(c_{0}\right)$, then

$$
\|P\| \geqq \frac{3 \alpha-\beta}{\alpha-\beta} .
$$

Proof. Given $0<\epsilon<\alpha / 2$, there exists a positive integer $N$ such that $x_{N}>\alpha-\epsilon$ and such that $\left|y_{j}\right|<\epsilon$ and $\beta-\epsilon<x_{j}<\alpha+\epsilon$ for all $i \geqq N$. Let $z=\left(z_{j}\right)$ be the element of $(c)$ such that

$$
z_{j}=\left\{\begin{array}{cl}
-x_{j} & \text { if } j<N, \\
\frac{\beta-3 \alpha}{2 \alpha} & \text { if } j=N, \\
-\frac{\alpha+\beta}{2} & \text { if } j>N .
\end{array}\right.
$$

2 The reader may be helped in the countable case by the remark that in Sobczyk's proof the matrices $\left\{\left\{k_{i j}(N)\right\}\right\}_{n}$ can be chosen so that if $n<n^{\prime}$, then $\left\{\left\{k_{i j}(N)\right\}\right\}_{n}$ is a submatrix of $\left\{\left\{k_{i j}(N)\right\}\right\}_{n^{\prime}}$. Since completing this paper, the author has learned from D. W. Dean of an alternative proof of Sobczyk's theorem and of Theorem 2.2 of the present paper. 
Then $P(z+x)=z+P x$. We observe that

$$
\|z+P x\| \geqq\left|z_{N}+y_{N}\right| \geqq \frac{3 \alpha-\beta}{2 \alpha}(\alpha-\epsilon)-\epsilon>0,
$$

and that

$$
\|z+x\|=\sup _{j}\left|z_{j}+x_{j}\right|
$$

$$
\leqq \frac{\alpha-\beta}{2}+\epsilon
$$

Now $\|P\| \geqq\|P(z+x)\| /\|z+x\|$, and it follows, since $\epsilon$ may be arbitrarily small, that (3.1) holds.

CoROLlary 3.2. If $B$ is any subspace of $(m)$ which properly contains (c) and if $P$ is any projection of $B$ onto $(c)$, then $\|P\| \geqq 2$.

Proof. There exists $y \neq 0$ in $B$ such that $P y=0$. Either $y$ or $-y$ must satisfy the hypothesis on $x$ in Theorem 3.1 , and since $B \supseteq(c)$ $+(y)$,

$$
\|P\| \geqq \frac{3 \alpha-\beta}{\alpha-\beta}=2+\frac{\alpha+\beta}{\alpha-\beta} \geqq 2 .
$$

Theorem 3.3. If $x$ is an element of $(m)$ but not of $(c)$, then

$$
\min \{\|P\|: P \text { is a projection of }(c)+(x) \text { onto }(c)\}=2 \text {. }
$$

Proof. It is trivial to find an element $y \in(c)$ such that $z=\left(z_{j}\right)$ $=x+y$ has the property that $\|z\|=\lim \sup _{j} z_{j}=-\lim \inf _{j} z_{j}$. Let $P$ be the projection of $(c)+(x)$ onto $(c)$ such that $P z=0$. Then for any real number $t$ and any $w \in(c)$,

$$
\|P(t z+w)\|=\|w\| \leqq\|t z+w\|+\|t z\| \leqq 2\|t z+w\|,
$$

which with Corollary 3.2 implies (3.6).

4. We now use Theorem 3.1 to show that the number 3 which appears in Theorem 2.2 cannot be lessened.

Given any integer $n \geqq 2$ let $B_{n}$ be the subspace of $(m)$ defined by

$$
B_{n}=\left\{y=\left(y_{j}\right): \lim _{t} y_{i+n t} \text { exists for } i=1, \cdots, n\right\} \text {. }
$$

For $i=1, \cdots, n$ let $x_{i}=\left(x_{i j}\right)$ be the element of $B_{n}$ such that $x_{i j}=1$ if $j \equiv i(\bmod n)$ and $x_{i j}=0$ otherwise. Then every $y \in B_{n}$ can be expressed uniquely in the form 


$$
y=z+\sum_{i=1}^{n} t_{i} x_{i}
$$

where $z \in\left(c_{0}\right)$ and $t_{1}, \cdots, t_{n}$ are real numbers.

THEOREM 4.1. For $n \geqq 2$,

(4.3) $\min \left\{\|P\|: P\right.$ is a projection of $B_{n}$ onto $\left.(c)\right\}=3-2 n^{-1}$.

Proof. Let $P_{n}$ be the projection of $B_{n}$ onto $(c)$ defined by

$$
P_{n} y=\left(w_{j}\right)=z+\sum_{i=1}^{n} t_{i} n^{-1} e .
$$

For $i_{0}=1, \cdots, n$ and $t \geqq 0$,

$$
\begin{aligned}
\left|w_{i_{0}+n t}\right| & =\left|y_{i_{0}+n t}+\sum_{i=1}^{n} t_{i}\left(n^{-1}-x_{i, i_{0}+n t}\right)\right| \\
& \leqq\left|y_{i_{0}+n t}\right|+\left(1-n^{-1}\right)\left|t_{i_{0}}\right|+n^{-1} \sum_{i=1 ; i \neq i_{0}}^{n}\left|t_{i}\right| \\
& \leqq\left(3-2 n^{-1}\right)|| y||,
\end{aligned}
$$

since $\left|t_{i}\right| \leqq\|y\|(i=1, \cdots, n)$. Thus, $\left\|P_{n}\right\| \leqq 3-2 n^{-1}$.

If on the other hand $P$ is any projection of $B_{n}$ onto $(c)$ and if $u_{i}=\left(u_{i j}\right)=P x_{i}$, then the limit $v_{i}=\lim _{j} u_{i j}$ exists. Since $P e=e$, it must be true that $\sum_{i=1}^{n} v_{i}=1$. Hence, there must exist some $i_{0}\left(1 \leqq i_{0} \leqq n\right)$ such that $v_{i_{0}} \leqq n^{-1}$. Letting $x=x_{i_{0}}-v_{i_{0}} e$, we see that $x$ satisfies the hypothesis of Theorem 3.1 with $\alpha=1-v_{i_{0}}, \beta=-v_{i_{0}}$. It follows that $\|P\| \geqq 3-2 v_{i_{0}} \geqq 3-2 n^{-1}$, and the theorem is proved.

Corollary 4.2. The space $B=\cup_{n=1}^{\infty} B_{n}$ is a separable subspace of (m) such that

$$
\min \{\|P\|: P \text { is a projection of } B \text { onto }(c)\}=3 .
$$

Proof. It is clear that $B$ is a separable subspace of $(m)$. If $P$ is a projection of $B$ onto $(c)$, then $\|P\| \geqq\left\|P \mid B_{n}\right\| \geqq 3-2 n^{-1}$ for all $n$, which with Theorem 2.2 implies (4.6).

REMARK 4.3. We exhibit finally a subspace $S$ of countably infinite dimension such that $S \cap(c)=\{0\}$, for which there does exist a projection of norm 2 of $S+(c)$ onto $(c)$. Let $S$ contain all finite linear combinations of $y_{1}, y_{2}, \cdots$ where $y_{i}=\left(y_{i j}\right)$ is defined by

$$
y_{i j}=\left\{\begin{aligned}
1 & \text { if } j \equiv 2^{i}\left(\bmod 2^{i}\right), \\
-1 & \text { if } j \equiv\left(2^{i}-1\right)\left(\bmod 2^{i}\right), \\
0 & \text { otherwise. }
\end{aligned}\right.
$$

Let $P$ be the projection of $S+(c)$ onto $(c)$ such that $P y_{i}=0$ for all 
$i$. If $z=\sum_{i=1}^{n} t_{i} y_{i}$, then $z$ has the properties of the $z$ in the proof of Theorem 3.3, and it follows that $\|P\|=2$.

\section{REFERENCES}

1. R. S. Phillips, On linear transformations, Trans. Amer. Math. Soc. vol. 48 (1940) pp. 516-541.

2. A. Sobczyk, Projection of the space $(m)$ on its subspace $\left(c_{0}\right)$, Bull. Amer. Math. Soc. vol. 47 (1941) pp. 938-947.

UNIVERSITY OF TENNESSEE AND

Princeton University

\section{EXPANSIONS OF PARABOLIC WAVE FUNCTIONS}

\section{Y. ALAVI ${ }^{1}$ AND C. P. WELLS}

1. Introduction. The main result of this paper is an expansion of parabolic wave functions in a series of spherical wave functions with coefficients expressed in terms of Pasternack's functions. The series can be inverted, giving spherical wave functions as integrals of parabolic wave functions. Special cases include an expansion due to Hochstadt [1] and the limiting case where the wave functions become potential functions. We also give a new derivation of the bilinear generating function in the continuous case for the parabolic wave functions.

We call either $\psi(\xi, \lambda) \psi(\eta,-\lambda) e^{i \mu \phi}$, or simply $\psi(\xi, \lambda) \psi(\xi,-\lambda)$, where $\psi(\xi, \lambda)$ satisfies

$$
\xi^{2} \frac{d^{2} z}{d \xi^{2}}+\xi \frac{d z}{d \xi}+\left(k^{2} \xi^{4}+\lambda \xi^{2}-\mu^{2}\right) z=0,
$$

a parabolic wave function. The solution of (1) in which we are interested is given by

$$
\psi(\xi, \lambda)=\xi^{\mu} \exp \left(i k \xi^{2} / 2\right)_{1} F_{1}\left((w+\mu+1) / 2 ; \mu+1 ;-i k \xi^{2}\right),
$$

or in Whittaker's notation

$$
\psi(\xi, \lambda)=(-i k)^{-\mu / 2}\left(-i k \xi^{2}\right)^{-1 / 2} M_{-w / 2, \mu / 2}\left(-i k \xi^{2}\right),
$$

where $\lambda$ and $k$ are real and $w=-i \lambda / 2 k$. In physical applications $\mu$ is often an integer since then $\psi(\xi, \lambda)$ is regular and single valued

Presented to the Society, January 22, 1959; received by the editors November 17, 1958 and, in revised form, February 2, 1959.

1 The first named author is at Western Michigan University at the present time. 